\title{
Tau Decays into Three Charged Leptons and Two Neutrinos
}

M. S. Alam, ${ }^{1}$ I. J. Kim, ${ }^{1}$ Z. Ling, ${ }^{1}$ A. H. Mahmood, ${ }^{1}$ J. J. O’Neill, ${ }^{1}$ H. Severini, ${ }^{1}$ C. R. Sun, ${ }^{1}$ S. Timm, ${ }^{1}$ F. Wappler, ${ }^{1}$ J.E. Duboscq, ${ }^{2}$ R. Fulton, ${ }^{2}$ D. Fujino, ${ }^{2}$ K. K. Gan,${ }^{2}$ K. Honscheid, ${ }^{2}$ H. Kagan,${ }^{2}$ R. Kass, ${ }^{2}$ J. Lee, ${ }^{2}$ M. Sung, ${ }^{2}$ C. White, ${ }^{2}$ R. Wanke, ${ }^{2}$ A. Wolf, ${ }^{2}$ M. M. Zoeller, ${ }^{2}$ X. Fu, ${ }^{3}$ B. Nemati, ${ }^{3}$ S. J. Richichi, ${ }^{3}$ W. R. Ross, ${ }^{3}$ P. Skubic, ${ }^{3}$ M. Wood, ${ }^{3}$ M. Bishai ${ }^{4}$ J. Fast, ${ }^{4}$ E. Gerndt, ${ }^{4}$ J. W. Hinson, ${ }^{4}$ T. Miao, ${ }^{4}$ D. H. Miller, ${ }^{4}$ M. Modesitt, ${ }^{4}$ E. I. Shibata, ${ }^{4}$ I. P. J. Shipsey, ${ }^{4}$ P. N. Wang, ${ }^{4}$ L. Gibbons,${ }^{5}$ S. D. Johnson, ${ }^{5}$ Y. Kwon, ${ }^{5}$ S. Roberts, ${ }^{5}$ E. H. Thorndike, ${ }^{5}$ C. P. Jessop, ${ }^{6}$ K. Lingel, ${ }^{6}$ H. Marsiske, ${ }^{6}$ M. L. Perl, ${ }^{6}$ S. F. Schaffner, ${ }^{6}$ R. Wang, ${ }^{6}$ T. E. Coan,${ }^{7}$ J. Dominick, ${ }^{7}$ V. Fadeyev, ${ }^{7}$ I. Korolkov, ${ }^{7}$ M. Lambrecht, ${ }^{7}$ S. Sanghera, ${ }^{7}$ V. Shelkov,${ }^{7}$ R. Stroynowski, ${ }^{7}$ I. Volobouev, ${ }^{7}$ G. Wei, ${ }^{7}$ M. Artuso, ${ }^{8}$ A. Efimov, ${ }^{8}$ M. Gao, ${ }^{8}$ M. Goldberg, ${ }^{8}$ D. He ${ }^{8}$ N. Horwitz,${ }^{8}$ S. Kopp,${ }^{8}$ G. C. Moneti, ${ }^{8}$ R. Mountain, ${ }^{8}$ Y. Mukhin, ${ }^{8}$ S. Playfer, ${ }^{8}$ T. Skwarnicki, ${ }^{8}$ S. Stone,${ }^{8}$ X. Xing, ${ }^{8}$ J. Bartelt, ${ }^{9}$ S. E. Csorna,,${ }^{9}$ V. Jain,${ }^{9}$ S. Marka, ${ }^{9}$ D. Gibaut, ${ }^{10}$ K. Kinoshita, ${ }^{10}$ P. Pomianowski, ${ }^{10}$ S. Schrenk, ${ }^{10}$ B. Barish,,${ }^{11}$ M. Chadha, ${ }^{11}$ S. Chan, ${ }^{11}$ G. Eigen, ${ }^{11}$ J. S. Miller, ${ }^{11}$ C. O' Grady, ${ }^{11}$ M. Schmidtler, ${ }^{11}$ J. Urheim, ${ }^{11}$ A. J. Weinstein, ${ }^{11}$ F. Würthwein, ${ }^{11}$ D. M. Asner, ${ }^{12}$ M. Athanas, ${ }^{12}$ D. W. Bliss, ${ }^{12}$ W. S. Brower, ${ }^{12}$ G. Masek, ${ }^{12}$ H. P. Paar, ${ }^{12}$ J. Gronberg, ${ }^{13}$ C. M. Korte, ${ }^{13}$ R. Kutschke, ${ }^{13}$ S. Menary, ${ }^{13}$ R. J. Morrison, ${ }^{13}$ S. Nakanishi, ${ }^{13}$ H. N. Nelson, ${ }^{13}$ T. K. Nelson, ${ }^{13}$ C. Qiao, ${ }^{13}$ J. D. Richman, ${ }^{13}$ D. Roberts, ${ }^{13}$ A. Ryd, ${ }^{13}$ H. Tajima, ${ }^{13}$ M. S. Witherell, ${ }^{13}$ R. Balest, ${ }^{14}$ K. Cho, ${ }^{14}$ W. T. Ford,${ }^{14}$ M. Lohner, ${ }^{14}$ H. Park, ${ }^{14}$ P. Rankin, ${ }^{14}$ J. Roy, ${ }^{14}$ J. G. Smith, ${ }^{14}$ J. P. Alexander, ${ }^{15}$ C. Bebek, ${ }^{15}$ B. E. Berger, ${ }^{15}$ K. Berkelman, ${ }^{15}$ K. Bloom,,${ }^{15}$ D. G. Cassel, ${ }^{15}$ H. A. Cho, ${ }^{15}$

D. M. Coffman, ${ }^{15}$ D. S. Crowcroft,${ }^{15}$ M. Dickson, ${ }^{15}$ P. S. Drell, ${ }^{15}$ D. J. Dumas, ${ }^{15}$ R. Ehrlich, ${ }^{15}$ R. Elia,,${ }^{15}$ P. Gaidarev, ${ }^{15}$ R. S. Galik, ${ }^{15}$ B. Gittelman, ${ }^{15}$ S. W. Gray, ${ }^{15}$ D. L. Hartill, ${ }^{15}$ B. K. Heltsley, ${ }^{15}$ C. D. Jones, ${ }^{15}$ S. L. Jones,${ }^{15}$ J. Kandaswamy, ${ }^{15}$ N. Katayama, ${ }^{15}$ P. C. Kim,,${ }^{15}$ D. L. Kreinick, ${ }^{15}$ T. Lee, ${ }^{15}$ Y. Liu, ${ }^{15}$ G. S. Ludwig, ${ }^{15}$ J. Masui, ${ }^{15}$ J. Mevissen, ${ }^{15}$ N. B. Mistry, ${ }^{15}$ C. R. Ng,${ }^{15}$ E. Nordberg, ${ }^{15}$ J. R. Patterson, ${ }^{15}$ D. Peterson, ${ }^{15}$ D. Riley, ${ }^{15}$ A. Soffer, ${ }^{15}$ C. Ward, ${ }^{15}$ P. Avery, ${ }^{16}$ A. Freyberger,${ }^{16}$ C. Prescott, ${ }^{16}$ S. Yang,,${ }^{16}$ J. Yelton,,${ }^{16}$ G. Brandenburg,,${ }^{17}$ R. A. Briere,${ }^{17}$ D. Cinabro, ${ }^{17}$ T. Liu, ${ }^{17}$ M. Saulnier,${ }^{17}$ R. Wilson, ${ }^{17}$ H. Yamamoto, ${ }^{17}$ T. E. Browder,${ }^{18}$ F. Li, ${ }^{18}$ J. L. Rodriguez, ${ }^{18}$ T. Bergfeld, ${ }^{19}$ B. I. Eisenstein, ${ }^{19}$ J. Ernst, ${ }^{19}$ G. E. Gladding, ${ }^{19}$ G. D. Gollin, ${ }^{19}$ M. Palmer, ${ }^{19}$ M. Selen, ${ }^{19}$ J. J. Thaler, ${ }^{19}$ K. W. Edwards, ${ }^{20}$ K. W. McLean, ${ }^{20}$ M. Ogg,${ }^{20}$ A. Bellerive, ${ }^{21}$ D. I. Britton, ${ }^{21}$ R. Janicek, ${ }^{21}$ D. B. MacFarlane, ${ }^{21}$ P. M. Patel, ${ }^{21}$ B. Spaan, ${ }^{21}$ A. J. Sadoff,${ }^{22}$ R. Ammar, ${ }^{23}$ P. Baringer, ${ }^{23}$ A. Bean, ${ }^{23}$ D. Besson, ${ }^{23}$ D. Coppage, ${ }^{23}$ N. Copty, ${ }^{23}$ R. Davis, ${ }^{23}$ N. Hancock, ${ }^{23}$ S. Kotov, ${ }^{23}$ I. Kravchenko, ${ }^{23}$ N. Kwak, ${ }^{23}$ Y. Kubota, ${ }^{24}$ M. Lattery, ${ }^{24}$ J. K. Nelson, ${ }^{24}$ S. Patton, ${ }^{24}$ R. Poling, ${ }^{24}$ T. Riehle, ${ }^{24}$ and V. Savinov ${ }^{24}$

\author{
(CLEO Collaboration) \\ ${ }^{1}$ State University of New York at Albany, Albany, New York 12222 \\ ${ }^{2}$ The Ohio State University, Columbus, Ohio 43210 \\ ${ }^{3}$ University of Oklahoma, Norman, Oklahoma 73019 \\ ${ }^{4}$ Purdue University, West Lafayette, Indiana 47907 \\ ${ }^{5}$ University of Rochester, Rochester, New York 14627 \\ ${ }^{6}$ Stanford Linear Accelerator Center, Stanford University, Stanford, California 94309 \\ ${ }^{7}$ Southern Methodist University, Dallas, Texas 75275 \\ ${ }^{8}$ Syracuse University, Syracuse, New York 13244 \\ ${ }^{9}$ Vanderbilt University, Nashville, Tennessee 37235 \\ ${ }^{10}$ Virginia Polytechnic Institute and State University, Blacksburg, Virginia 24061 \\ ${ }^{11}$ California Institute of Technology, Pasadena, California 91125 \\ ${ }^{12}$ University of California, San Diego, La Jolla, California 92093 \\ ${ }^{13}$ University of California, Santa Barbara, California 93106 \\ ${ }^{14}$ University of Colorado, Boulder, Colorado 80309-0390 \\ ${ }^{15}$ Cornell University, Ithaca, New York 14853 \\ ${ }^{16}$ University of Florida, Gainesville, Florida 32611 \\ ${ }^{17}$ Harvard University, Cambridge, Massachusetts 02138 \\ ${ }^{18}$ University of Hawaii at Manoa, Honolulu, Hawaii 96822 \\ ${ }^{19}$ University of Illinois, Champaign-Urbana, Illinois 61801 \\ ${ }^{20}$ Carleton University, Ottawa, Ontario, Canada K1S 5B6 \\ and the Institute of Particle Physics, Montréal, Québec, Canada \\ ${ }^{21}$ McGill University, Montréal, Québec, Canada H3A 278 \\ and the Institute of Particle Physics, Montréal, Québec, Canada \\ ${ }^{22}$ Ithaca College, Ithaca, New York 14850 \\ ${ }^{23}$ University of Kansas, Lawrence, Kansas 66045
}


${ }^{24}$ University of Minnesota, Minneapolis, Minnesota 55455

(Received 22 November 1995; revised manuscript received 8 February 1996)

We search for the radiative leptonic tau decays $\tau \rightarrow e e^{+} e^{-} \nu_{\tau} \nu_{e}$ and $\tau \rightarrow \mu e^{+} e^{-} \nu_{\tau} \nu_{\mu}$ using $3.60 \mathrm{fb}^{-1}$ of data collected by the CLEO-II experiment at the Cornell Electron Storage Ring. We present a first observation of the $\tau \rightarrow e e^{+} e^{-} \nu_{\tau} \nu_{e}$ process. For this channel we measure the branching fraction $B\left(\tau \rightarrow e e^{+} e^{-} \nu_{\tau} \nu_{e}\right)=\left(2.7_{-1.1-0.4-0.3}^{+1.5+0.4+0.1}\right) \times 10^{-5}$. An upper limit is established for the second channel: $B\left(\tau \rightarrow \mu e^{+} e^{-} \nu_{\tau} \nu_{\mu}\right)<3.2 \times 10^{-5}$ at $90 \%$ C.L. Both results are consistent with the rates expected from standard model predictions.

PACS numbers: 13.35.Dx

Tau decays into three charged leptons and two neutrinos are allowed processes in the standard model. They proceed via emission of a virtual photon with subsequent internal conversion into a pair of electrons or muons. Two Feynman diagrams provide the dominant contribution to the decay rate. They are shown in Fig. 1 for the $\tau^{-} \rightarrow \mu^{-} e^{+} e^{-} \nu_{\tau} \bar{\nu}_{\mu}$ decay. The contribution of a third diagram, with a virtual photon emitted from the $W$ boson, is heavily suppressed by the $W$ propagator. For tau decays with two identical charged leptons in the final state, two additional exchange diagrams are involved. Branching fractions for these processes have been recently calculated by Dicus and Vega [1] and are listed in Table I. The branching fractions for tau decays with a virtual photon conversion into two muons, $\tau \rightarrow e \mu^{+} \mu^{-} \nu_{\tau} \nu_{e}$ and $\tau \rightarrow$ $\mu \mu^{+} \mu^{-} \nu_{\tau} \nu_{\mu}$, are expected to be at the level of $10^{-7}$, too small to be observed in existing data. On the other hand, the expected branching fractions for $\tau \rightarrow e e^{+} e^{-} \nu_{\tau} \nu_{e}$ and $\tau \rightarrow \mu e^{+} e^{-} \nu_{\tau} \nu_{\mu}$ are at the level of $10^{-5}$, which is comparable to the sensitivity reached in a recent search for neutrinoless tau decays into three charged particles [2]. In this Letter, we report on a follow-up study in which we have searched for these two decays. Radiative tau decays into a muon, two neutrinos, and a photon without internal conversion have been previously observed [3].

The data used in this analysis were collected with the CLEO-II detector at the Cornell Electron Storage Ring (CESR), in which tau leptons are produced in pairs in $e^{+} e^{-}$collisions. They correspond to an integrated luminosity of about $3.60 \mathrm{fb}^{-1}$ and the number of produced tau pairs, $N_{\tau \tau}$, is $(3.28 \pm 0.05) \times 10^{6}$. About $60 \%$ of the events were obtained at the $\Upsilon(4 S)$ resonance $(\sqrt{s} \simeq$ $10.59 \mathrm{GeV}$ ) while the rest were obtained at energies approximately $60 \mathrm{MeV}$ lower. We use information from a 67-layer tracking system which also provides specific ionization measurements $(d E / d x)$, time-of-flight scintillation counters, and a 7800-crystal CsI calorimeter. These elements are inside a $1.5 \mathrm{~T}$ superconducting solenoidal magnet whose iron yoke also serves as a hadron absorber for a muon identification system. A detailed description of the apparatus can be found in Ref. [4].

In order to obtain a Monte Carlo event generator which we needed to design the event selection procedure and to estimate the detector acceptance, we performed the calculation of the relevant matrix elements using the sym- bolic manipulation program FORM [5]. No tau polarization effects or higher order radiative corrections were taken into account. To check our generator, we calculated the branching fraction for the known five lepton decay of the muon, $\mu \rightarrow e e e \nu_{\mu} \nu_{e}$. The result, listed in Table I, is consistent with the calculation of Dicus and Vega [1] and with an earlier estimate $B\left(\mu \rightarrow e e e \nu_{\mu} \nu_{e}\right)=(3.54 \pm$ $0.09) \times 10^{-5}$ by Bardin, Istatkov, and Mitsel'makher [6]. It also agrees with the measurement $B\left(\mu \rightarrow e e e \nu_{\mu} \nu_{e}\right)=$ $(3.4 \pm 0.4) \times 10^{-5}$ by the SINDRUM Collaboration [7]. For tau decays our branching fraction estimates are (67)\% higher than those of Ref. [1]. We generated 100000 $\tau \rightarrow \mu e^{+} e^{-} \nu_{\tau} \nu_{\mu}$ and $60000 \tau \rightarrow e e^{+} e^{-} \nu_{\tau} \nu_{e}$ Monte Carlo decays to study their kinematical properties and the response of the detector. The KORALB/TAUOLA program package [8] was used to simulate the tau-pair production and the decay of the other tau in the event. Detector signals were simulated by the standard CLEO-II simulation program [9].

To extract from our data tau decays into three charged leptons and two neutrinos, we search for events where one tau decays into a single charged particle (1-prong decay) and the other tau decays into three charged particles (3prong decay). The 3-prong decay is a signal candidate and the 1-prong is an allowed tau decay with one charged particle, zero or more photons and at least one neutrino in the final state. For each candidate event we require four well-reconstructed charged particle tracks with zero total charge. The most isolated track must be separated by at least $90^{\circ}$ from all other tracks. We also reject events with photons with energies larger than $60 \mathrm{MeV}$ on the 3-prong side.

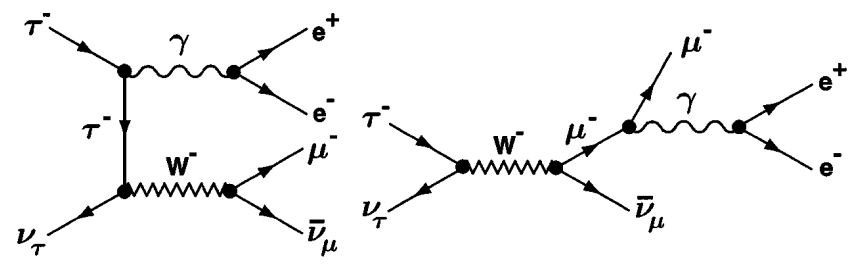

FIG. 1. Feynman diagrams for the $\tau^{-} \rightarrow \mu^{-} e^{+} e^{-} \nu_{\tau} \bar{\nu}_{\mu}$ process. 
TABLE I. Calculated $\tau$ and $\mu$ branching fractions. Errors given here are due to inaccuracies in numerical integration only.

\begin{tabular}{lcr}
\hline \hline Channel & Dicus and Vega & Our calculation \\
\hline$\tau \rightarrow e e e \nu_{\tau} \nu_{e}$ & $(4.15 \pm 0.06) \times 10^{-5}$ & $(4.457 \pm 0.006) \times 10^{-5}$ \\
$\tau \rightarrow \mu e e \nu_{\tau} \nu_{\mu}$ & $(1.97 \pm 0.02) \times 10^{-5}$ & $(2.089 \pm 0.003) \times 10^{-5}$ \\
$\tau \rightarrow e \mu \mu \nu_{\tau} \nu_{e}$ & $(1.257 \pm 0.003) \times 10^{-7}$ & $(1.347 \pm 0.002) \times 10^{-7}$ \\
$\tau \rightarrow \mu \mu \mu \nu_{\tau} \nu_{\mu}$ & $(1.190 \pm 0.002) \times 10^{-7}$ & $(1.276 \pm 0.004) \times 10^{-7}$ \\
$\tau \rightarrow e e e \nu_{\mu} \nu_{e}$ & $(3.60 \pm 0.02) \times 10^{-5}$ & $(3.605 \pm 0.005) \times 10^{-5}$ \\
\hline \hline
\end{tabular}

Substantial background suppression comes from lepton identification on the 3-prong side. In the $\tau \rightarrow$ $\mu e^{+} e^{-} \nu_{\tau} \nu_{\mu}$ channel we require a muon candidate with momentum less than $2.2 \mathrm{GeV} / c$ to pass through at least three hadronic absorption lengths of iron and through at least five absorption lengths if its momentum is greater than $2.2 \mathrm{GeV} / c$. For such muon candidates, the energy deposited in the CsI calorimeter, $E_{c}$, must be compatible with that expected for a minimum ionizing particle: $0.1<E_{c}<0.5 \mathrm{GeV}$. We also require that the charge of the muon candidate is opposite to that of the 1-prong track. For electron identification, we rely mostly on the $d E / d x$ measurement in the drift chamber. It must differ from the expected value by less than 3 standard deviations, $\sigma$. If there is a time-of-flight measurement, we require that it is compatible with the electron hypothesis within $3 \sigma$, and if the electron candidate is fast enough so that its energy losses in the inner layers of the detector material can be neglected then we require that its $E_{c}$ is about the same as expected from its momentum value, $p_{e}$, measured in the drift chamber: $0.8<E_{c} / p_{e}<1.1$. This last requirement must be satisfied for at least one electron candidate in the $\tau \rightarrow e e^{+} e^{-} \nu_{\tau} \nu_{e}$ channel. In order to suppress a strong $e^{+} e^{-} \rightarrow e^{+} e^{-} e^{+} e^{-}$background we also require that in this channel the 1-prong particle is not consistent with being an electron. It must either pass through three absorption lengths of the muon filter or have a $E_{c} / p$ ratio incompatible with an electron hypothesis. The radiative muon pair background $e^{+} e^{-} \rightarrow \mu^{+} \mu^{-} e^{+} e^{-}$in the $\tau \rightarrow \mu e^{+} e^{-} \nu_{\tau} \nu_{\mu}$ channel is reduced by the requirement that the 1-prong particle is not identified as a muon.

The main sources of background left after lepton identification are as follows: low multiplicity $e^{+} e^{-} \rightarrow q \bar{q}$ events, 2-photon processes, especially the $e^{+} e^{-} \rightarrow \tau^{+} \tau^{-} e^{+} e^{-}$reaction which can result in a similar final state, radiative Bhabha, $\mu$ pairs, and radiative leptonic decays $\tau \rightarrow l \nu_{\tau} \nu_{l} \gamma(l$ stands for $e$ or $\mu$ ) with subsequent $\gamma \rightarrow e^{+} e^{-}$conversion in the detector material, tau decays into three hadrons and a neutrino where all hadrons are misidentified as leptons, and finally tau decays into $\rho \nu_{\tau}$ with subsequent $\rho \rightarrow \pi \pi^{0}$, $\pi^{0} \rightarrow e^{+} e^{-} \gamma$ decays, where the $\gamma$ escapes detection and the $\pi$ is misidentified as a lepton.
In order to suppress the non-tau background, we require undetected neutrinos to be present by selecting events with large missing energy, $E_{\text {miss }}>1.5 \mathrm{GeV}$, and with a large value of total transverse momentum of the charged particles with respect to the beam direction, $p_{t}>$ $150 \mathrm{MeV} / c$. The $\tau \rightarrow 3 h \nu_{\tau}$ decays contribute to the background in our analysis due to a rather large branching fraction, about $8.4 \%$ [10], and a few percent probability for pions to fake leptons. To suppress this background, we estimate the probability that all electron candidates in the event are pions using $d E / d x$ measurements. We define the quantities

$$
\begin{aligned}
\kappa_{2} & =\frac{P_{e^{+}} P_{e^{-}}}{P_{e^{+}} P_{e^{-}}+P_{\pi^{+}} P_{\pi^{-}}}, \\
\kappa_{3} & =\frac{P_{e} P_{e^{+}} P_{e^{-}}}{P_{e} P_{e^{+}} P_{e^{-}}+P_{\pi} P_{\pi^{+}} P_{\pi^{-}}}
\end{aligned}
$$

for the $\tau \rightarrow \mu e^{+} e^{-} \nu_{\tau} \nu_{\mu}$ and $\tau \rightarrow e e^{+} e^{-} \nu_{\tau} \nu_{e}$ channels, respectively, where $P_{e}=(2 \pi)^{-1 / 2} \exp \left(-\sigma_{e}^{2} / 2\right)$ and $P_{\pi}=(2 \pi)^{-1 / 2} \exp \left(-\sigma_{\pi}^{2} / 2\right)$. Here, $\sigma_{e}$ and $\sigma_{\pi}$ are the numbers of standard deviations of the measured specific ionization from that expected for an electron and a pion. $\kappa_{2}$ and $\kappa_{3}$ characterize the purity of the sample from a contamination with events with pions faking electrons. We require $\kappa_{2}$ and $\kappa_{3}$ to be greater than 0.97 .

We check for photon conversions in our data sample by reconstructing a possible conversion point. At such a point, the $e^{+}$and $e^{-}$tracks should be parallel in the transverse plane perpendicular to the beam axis. We require that the distance from this point to the beam axis must be less than $2 \mathrm{~cm}$. This suppresses photon conversions because the closest region where photons can convert in the detector material is the beam pipe at a radius of $3.5 \mathrm{~cm}$ from the beam axis. In the $\tau \rightarrow$ $e e^{+} e^{-} \nu_{\tau} \nu_{e}$ channel this requirement must be satisfied for both $e^{+} e^{-}$combinations.

In the $\tau \rightarrow \mu e^{+} e^{-} \nu_{\tau} \nu_{\mu}$ and $\tau \rightarrow e e^{+} e^{-} \nu_{\tau} \nu_{e}$ processes the invariant mass of the three charged leptons tends to be small and thus at the interaction point their tracks are nearly parallel. This feature provides the possibility of differentiating these decays from the $\tau \rightarrow$ $\rho \nu_{\tau}, \rho \rightarrow \pi \pi^{0}, \pi^{0} \rightarrow e^{+} e^{-} \gamma$ process where the $\gamma$ escapes detection and the $\pi$ is misidentified as a lepton. The distribution of the sum of the cosines of the angles 


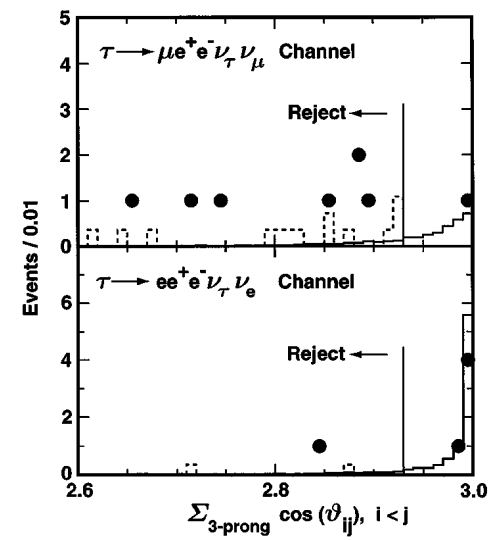

FIG. 2. Sum of cosines of the angles $\vartheta_{i j}$ between the 3-prong side tracks for the data (black circles), signal Monte Carlo (solid line), and generic tau Monte Carlo sample (dashed line) for the two channels studied. The signal Monte Carlo histograms are normalized to standard model theoretical predictions. The generic tau Monte Carlo histograms are normalized to the data luminosity. In this analysis we require $\sum_{i<j} \cos \vartheta_{i j}>2.93$ for both channels.

$\vartheta_{i j}$ between the 3-prong tracks is shown in Fig. 2 for the data, signal Monte Carlo events, and a sample of generic tau Monte Carlo events. We compare the distributions for the signal and generic tau Monte Carlo events and require $\sum_{i<j} \cos \vartheta_{i j}>2.93$ for both channels.

The signal efficiency, $\epsilon$, after application of all the selection requirements and accounting for tau pair tagging, is estimated from the Monte Carlo simulation to be $(2.7 \pm 0.1) \%$ for the $\tau \rightarrow e e^{+} e^{-} \nu_{\tau} \nu_{e}$ channel and $(1.9 \pm 0.1) \%$ for the $\tau \rightarrow \mu e^{+} e^{-} \nu_{\tau} \nu_{\mu}$ channel (statistical errors only). The main reason for such low efficiencies is a very soft momentum spectrum of the radiative electrons. With these estimates, our calculations predict that on average $7.8 \tau \rightarrow e e^{+} e^{-} \nu_{\tau} \nu_{e}$ and 2.6 $\tau \rightarrow \mu e^{+} e^{-} \nu_{\tau} \nu_{\mu}$ events will remain. In the data, five events satisfy all selection criteria in the $\tau \rightarrow e e^{+} e^{-} \nu_{\tau} \nu_{e}$ channel and one event in the $\tau \rightarrow \mu e^{+} e^{-} \nu_{\tau} \nu_{\mu}$ channel. Distributions of several kinematic variables for both the signal Monte Carlo and the selected data events are shown in Fig. 3 for the $\tau \rightarrow e e^{+} e^{-} \nu_{\tau} \nu_{e}$ channel. They indicate that the five remaining events in this channel are kinematically consistent with tau decays into three electrons and two neutrinos.

The remaining background from other tau decays is estimated by applying the same selection criteria to a sample of generic $e^{+} e^{-} \rightarrow \tau^{+} \tau^{-}$Monte Carlo events without the signal channels which is about 2.8 times larger than the data. No generic tau Monte Carlo events are accepted in either of the two channels. Thus, we estimate the background contribution from other tau decays to be less than 0.4 event at $68 \%$ confidence level (C.L.). We estimate the background from the $e^{+} e^{-} \rightarrow \tau^{+} \tau^{-} e^{+} e^{-}$ process using a sample of $6.6 \times 10^{5}$ Monte Carlo events (ten times larger than the data) generated with the DIAG36
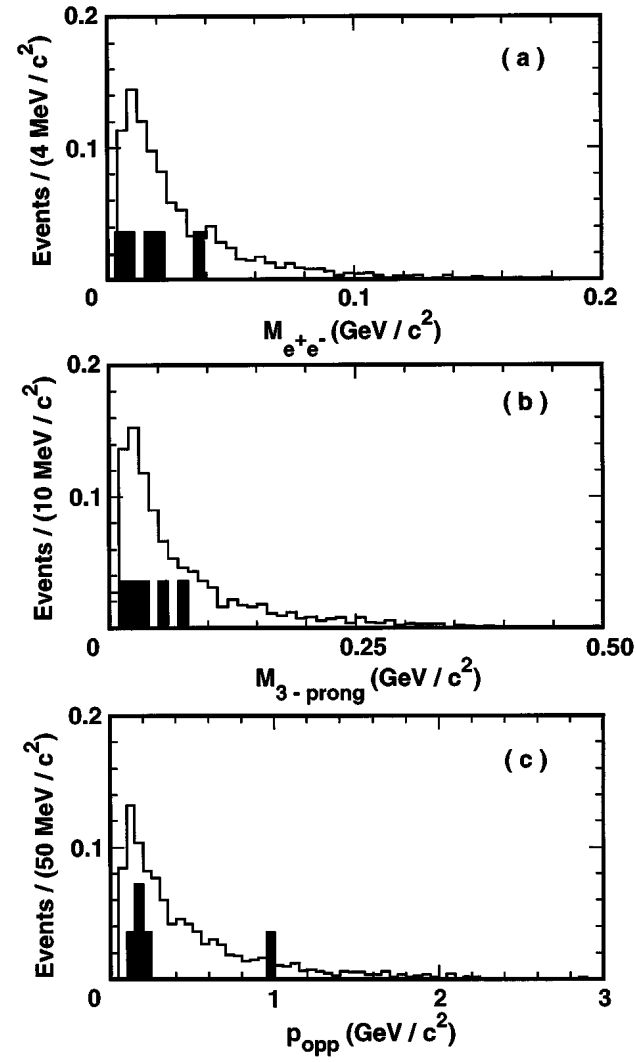

FIG. 3. Comparison of the kinematical distributions of the $\tau \rightarrow e e^{+} e^{-} \nu_{\tau} \nu_{e}$ Monte Carlo (solid line) and the data (shaded histogram) for events passing all selection requirements: (a) the $e^{+} e^{-}$invariant mass averaged over two possible combinations, $M_{e^{+} e^{-}}$, (b) the 3-prong invariant mass, $M_{3 \text {-prong, and (c) the }}$ momentum of the electron on the 3-prong side with the charge opposite to that of the parent tau, $p_{\mathrm{opp}}$. The normalization of the plots is arbitrary.

program [11]. From this source we expect on average 0.5 event in the $\tau \rightarrow \mu e^{+} e^{-} \nu_{\tau} \nu_{\mu}$ channel and 0.3 event in the $\tau \rightarrow e e^{+} e^{-} \nu_{\tau} \nu_{e}$ channel. No events satisfied our selection criteria in either of the two channels from samples of $e^{+} e^{-} \rightarrow B \bar{B}$ and continuum $\left(e^{+} e^{-} \rightarrow q \bar{q}\right.$, $q=u, d, s$, and $c$ ) Monte Carlo events which are larger than the data by factors of 2.6 and 1.2 , respectively. Kinematic properties of the $B \bar{B}$ and continuum events are very dissimilar to those of the signal, and we conclude that backgrounds from these sources are negligible. We expect no $e^{+} e^{-} \rightarrow e^{+} e^{-} e^{+} e^{-}$background in the $\tau \rightarrow$ $e e^{+} e^{-} \nu_{\tau} \nu_{e}$ channel after requiring that the 1-prong track must not be an electron. We checked this conclusion by looking at the $d E / d x$ measurements of the 1-prong tracks. These measurements favor the pion hypothesis over the electron one in all five remaining events. In addition, three of those events have a pair of photons on the 1-prong side with an invariant mass compatible with that of a $\pi^{0}$.

The main systematic errors in this study arise from uncertainties in our knowledge of the lepton identification efficiency and the reconstruction efficiency of slow tracks. 
Combined together, they are estimated to give an overall systematic error of $15 \%$. Thus, subtracting the expected background from $e^{+} e^{-} \rightarrow \tau^{+} \tau^{-} e^{+} e^{-}$events, our estimate of the $\tau \rightarrow e e^{+} e^{-} \nu_{\tau} \nu_{e}$ branching fraction is

$$
B\left(\tau \rightarrow e e^{+} e^{-} \nu_{\tau} \nu_{e}\right)=\left(2.7_{-1.1-0.4-0.3}^{+1.5+0.4+0.1}\right) \times 10^{-5},
$$

where the first errors account for statistical fluctuations and show a minimal $68 \%$ C.L. interval calculated according to Bayesian statistics with an assumption of a flat prior distribution [12], the second errors are due to systematic effects and the third set of errors reflects the uncertainty in our knowledge of background. Neglecting the second and third errors, our calculated value of $4.46 \times 10^{-5}$ is compatible with this result at $24 \%$ C.L. (we quote Bayesian confidence level here).

In the $\tau \rightarrow \mu e^{+} e^{-} \nu_{\tau} \nu_{\mu}$ channel we calculate an upper limit on the branching fraction according to a procedure described in Ref. [10] assuming an expected background of 0.5 event. As previously, we assign a systematic error of $15 \%$ to this result and increase the branching fraction limit by this amount. The resulting upper limit is

$$
B\left(\tau \rightarrow \mu e^{+} e^{-} \nu_{\tau} \nu_{\mu}\right)<3.2 \times 10^{-5} \text { at } 90 \% \text { C.L. }
$$

We gratefully acknowledge the effort of the CESR staff in providing us with excellent luminosity and running conditions. This work was supported by the National Science Foundation, the U.S. Department of Energy, the
Heisenberg Foundation, the Alexander von Humboldt Stiftung, the Natural Sciences and Engineering Research Council of Canada, and the A. P. Sloan Foundation.

[1] D. A. Dicus and R. Vega, Phys. Lett. B 338, 341 (1994).

[2] J. Bartelt et al., Phys. Rev. Lett. 73, 1890 (1994).

[3] D. Y. Wu et al., Phys. Rev. D 41, 2339 (1990).

[4] Y. Kubota et al., Nucl. Instrum. Methods Phys. Res., Sect. A 320, 66 (1992).

[5] J. A. M. Vermaseren, Report No. KEK-TH-326, 1992 (unpublished).

[6] D. Yu. Bardin, Ts. G. Istatkov, and G. B. Mitsel'makher, Sov. J. Nucl. Phys. 15, 161 (1972).

[7] W. Bertl et al., Nucl. Phys. B260, 1 (1985).

[8] S. Jadach and Z. Wa̧s, Comput. Phys. Commun. 64, 267 (1991); S. Jadach et al., Comput. Phys. Commun. 76, 361 (1993).

[9] Detector simulation is based on the GEANT software package: R. Brun et al., GEANT version 3.15, CERN Report No. DD/EE/84-1.

[10] Particle Data Group, L. Montanet et al., Phys. Rev. D 50, 1173 (1994).

[11] F. A. Berends, P.H. Daverveldt, and R. Kleiss, Comput. Phys. Commun. 40, 285 (1986).

[12] W.T. Eadie et al., Statistical Methods in Experimental Physics (North-Holland Publishing Company, Amsterdam, 1971), p. 213. 